\title{
FUNDAMENTAL GROUP AND PLURIDIFFERENTIALS ON COMPACT KÄHLER MANIFOLDS
}

\author{
YOHAN BRUNEBARBE \& FRÉDÉRIC CAMPANA
}

\begin{abstract}
A compact Kähler manifold $X$ is shown to be simplyconnected if its 'symmetric cotangent algebra' is trivial. Conjecturally, such a manifold should even be rationally connected. The relative version is also shown: a proper surjective connected holomorphic map $f: X \rightarrow S$ between connected manifolds induces an isomorphism of fundamental groups if its smooth fibres are as above, and if $X$ is Kähler.
\end{abstract}

\section{INTRODUCTION}

We shall show:

Theorem 1.1. Let $X$ be a connected compact Kähler manifold. Suppose that for all $p \geq 1$ and $k \geq 1$ there is no non-zero global section of the sheaf $S^{k} \Omega_{X}^{p}$. Then $X$ is simply connected].

This theorem refines a former result of [5] with the very same statement, but with $\otimes^{k} \Omega_{X}^{p}$ in place of $S^{k} \Omega_{X}^{p}$. The proof of 1.1 is obtained by refining the proof of [5], which rests on $L^{2}$-methods à la PoincaréAtiyah-Gromov.

The 'uniruledness conjecture' below implies easily (see $\$ 3$ ) that $X$ should, in fact, be rationally connected, hence simply-connected, by [3. Theorem 1.1 above permits to bypass this conjecture, as far as the fundamental group is concerned. It is usually quite easy to verify the vanishings of all $S^{k} \Omega_{X}^{p}$, while constructing sufficiently many rational curves requires the characteristic $p>0$ methods introduced by S. Mori, no characteristic zero proof being presently known.

The weaker assumption that $H^{0}\left(X, S^{k} \Omega_{X}^{1}\right)=\{0\}$ for every $k \geq$ 1 implies (see [2]) that all linear representations of the fundamental group $\pi_{1}(X) \rightarrow G L_{n}(K), K$ a field, have finite image. This raises the question of whether the condition $H^{0}\left(X, S^{k} \Omega_{X}^{1}\right)=\{0\}$ for every $k \geq 1$ might imply that $\pi_{1}(X)$ is finite, instead of trivial. Enriques

\footnotetext{
${ }^{1} \mathrm{By}$ a theorem of Kodaira, any $X$ as above is actually projective.
} 
surfaces (examples of general type also exist) indeed show that simpleconnectedness may then fail?

In contrast to the condition $H^{0}\left(X, S^{k} \Omega_{X}^{p}\right)=\{0\}$ for every $k \geq 1$ and $p \geq 1$, the condition $H^{0}\left(X, S^{k} \Omega_{X}^{1}\right)=\{0\}$ for every $k \geq 1$ does not seem however to have an even conjectural geometric interpretation in the frame of bimeromorphic classification of compact Kähler manifolds.

The theorem 1.1 above has a relative version, shown in section $\$$ below:

Corollary 1. Let $f: X \rightarrow S$ be a proper holomorphic map with connected fibres between connected complex manifolds. Assum that $X$ admits a Kähler metric, and that $f_{*}\left(S^{k}\left(\Omega_{X / S}^{p}\right)\right)=0$ for every $k \geq 1$ and $p \geq 1$. Then $f_{*}: \pi_{1}(X) \rightarrow \pi_{1}(S)$ is an isomorphism of groups.

Note that the conclusion of corollary 1 may fail for a projective morphism $f: X \rightarrow S$ with smooth fibres simply-connected, because of the possible presence of multiple fibres. Consider indeed an Enriques surface $Y$ and its $K 3$ universal cover $Y^{\prime} \rightarrow Y=Y^{\prime} / \mathbb{Z}_{2}$. Let $C \rightarrow \mathbb{P}^{1}=$ $C / \mathbb{Z}_{2}$ be the 2 -sheeted cover defined by a hyperelliptic curve $C$. Now let $X \rightarrow S:=\mathbb{P}^{1}$ be deduced from the first projection $X^{\prime}:=C \times Y^{\prime} \rightarrow C$ by taking the equivariant quotient by the involution $u \times v$ acting freely on $X^{\prime}, u$ and $v$ being the involutions on $Y^{\prime}$ and $C$ respectively deduced from the $\mathbb{Z}_{2}$ covers above. Here $S=\mathbb{P}^{1}$ is simply connected although $\pi_{1}(X)$ is a $\mathbb{Z}_{2}$ extension of $\pi_{1}(C)$ and the smooth fibres of $f$ are simplyconnected.

\section{Proof of theorem 1.1}

As in [5], the proof goes in two steps: show first that $\pi_{1}(X)$ is finite (this is the main step, established below), and then show, using Serre's covering trick, that $\pi_{1}(X)$ is in fact trivial.

We start by establishing this second step. Let $\pi: X^{\prime} \rightarrow X$ be a finite Galois étale cover of $X$ of group $G$ and degree $d$. The Euler characteristic of the structural sheaf of $X$

$$
\chi\left(X, \mathcal{O}_{X}\right):=\sum_{i=0}^{\operatorname{dim} X}(-1)^{i} \cdot h^{i}\left(X, \mathcal{O}_{X}\right)
$$

is equal to 1 , since by Serre's duality $h^{i}\left(X, \mathcal{O}_{X}\right)=h^{0}\left(X, \Omega_{X}^{i}\right)$, and the latter is zero for $i \neq 0$ by hypothesis.

\footnotetext{
${ }^{2}$ Hopf surfaces $X$ have $H^{0}\left(X, S^{k} \Omega_{X}^{p}\right)=\{0\}, \forall k>1, p>1$, showing that the Kähler assumption cannot be removed in 1.1. since $\pi_{1}(X) \cong \mathbb{Z}$.

${ }^{3}$ These hypothesis should imply that $f$ is projective, locally above $S$.
} 
Now, if $\omega \in H^{0}\left(X^{\prime}, \Omega_{X^{\prime}}^{i}\right)$, the product of the $g^{*} \omega$ for $g \in G$ defines an element of $H^{0}\left(X^{\prime}, S^{d} \Omega_{X^{\prime}}^{i}\right)$ invariant by the action of $G$. We obtain in this way a global section of $S^{d} \Omega_{X}^{i}$, which is non zero if $\omega$ is non zero. Thus it follows from the hypothesis that we must also have $\chi\left(X^{\prime}, \mathcal{O}_{X^{\prime}}\right)=1$.

From the multiplicativity of the Euler characteristic (see lemma 2.1 below), we get:

$$
1=\chi\left(X^{\prime}, \mathcal{O}_{X^{\prime}}\right)=d \cdot \chi\left(X, \mathcal{O}_{X}\right),
$$

and $d$ is then necessarly equal to 1 .

Lemma 2.1. Let $X^{\prime} \rightarrow X$ be a finite étale covering of degree $d$ of compact complex analytic spaces. Then

$$
\chi\left(X^{\prime}, \mathcal{O}_{X^{\prime}}\right)=d \cdot \chi\left(X, \mathcal{O}_{X}\right) .
$$

Proof. When $X$ is projective, an elementary proof due to Kleiman is given in [12, exemple 1.1.30. In general, it is an easy consequence of the theorem of Riemann-Roch-Hirzebruch, which is proved in [14 for compact complex analytic spaces.

. To complete the proof of theorem 1.1, we need to show that the fundamental group of $X$ is finite. Equivalently, we have to show the

Theorem 2.2. Let $X$ be a connected compact Kähler manifold with infinite fundamental group. Then there exists $p \geq 1$ and $k \geq 1$ such that $H^{0}\left(X, S^{k} \Omega_{X}^{p}\right) \neq\{0\}$.

Proof. Let $p: \tilde{X} \rightarrow X$ be the universal cover of $X$. The fundamental group $\Gamma:=\pi_{1}(X)$ acts on $\tilde{X}$. The choice of a Kähler metric on $X$ induces a complete Kähler metric on $\tilde{X}$. Denote by $\mathcal{H}_{(2)}^{k}(\tilde{X})$ the Hilbert space of $L^{2}$-harmonic complex-valued forms of degree $k$ on $\tilde{X}$. Recall that a $p$-form $\alpha$ is called harmonic if $\Delta \alpha=0$, where $\Delta:=d \circ d^{*}+d^{*} \circ d$ and $d^{*}:=-* \circ d \circ *$. Moreover, a $L^{2} p$-form $\alpha$ is harmonic if and only if $d \alpha=0$ and $d^{*} \alpha=0$ (the metric being complete), if and only if $\bar{\partial} \alpha=0$ and $\bar{\partial}^{*} \alpha=0$ (the metric being complete and Kähler), see [9].

The decomposition in types gives rise to a orthogonal sum

$$
\mathcal{H}_{(2)}^{k}(\tilde{X})=\bigoplus_{p+q=k} \mathcal{H}_{(2)}^{p, q}(\tilde{X})
$$

The space $\mathcal{H}_{(2)}^{p, 0}(\tilde{X})$ consists of the $L^{2}$-holomorphic $p$-forms on $\tilde{X}$.

\footnotetext{
${ }^{4}$ We shall only need the case when $X$ is a divisor with normal crossings in a complex Kähler manifold in the proof of corollary 1
} 
The Hilbert spaces $\mathcal{H}_{(2)}^{p, q}(\tilde{X})$ might be infinite dimensional. Nevertheless, using the isometric action of $\Gamma$ on them, one can associate to them a non-negative real number $\operatorname{dim}_{\Gamma}\left(\mathcal{H}_{(2)}^{p, q}(\tilde{X})\right)$ (cf. [1]). This number is zero if and only if $\mathcal{H}_{(2)}^{p, q}(\tilde{X})=\{0\}$.

By Atiyah's $L^{2}$-index theorem (cf. [1, 9]), we know that

$$
\chi\left(X, \mathcal{O}_{X}\right)=\chi_{(2)}\left(\tilde{X}, \mathcal{O}_{\tilde{X}}\right):=\sum_{q=0}^{\operatorname{dim} X}(-1)^{q} \cdot \operatorname{dim}_{\Gamma}\left(\mathcal{H}_{(2)}^{0, q}(\tilde{X})\right)
$$

Observe that there are no non-zero $L^{2}$-holomorphic functions on $\tilde{X}$. Indeed, the metric being complete, any harmonic function is closed, hence locally constant. By hypothesis $\tilde{X}$ is non-compact, and any constant $L^{2}$ function has to be zero.

Let us distinguish two cases. Suppose first that $\chi\left(X, \mathcal{O}_{X}\right)=0$. Since $\operatorname{dim} H^{0}\left(X, \mathcal{O}_{X}\right)=1$, Hodge symmetry shows that $H^{0}\left(X, \Omega_{X}^{p}\right) \neq\{0\}$, for some (odd) $p \geq 1$, and the theorem is proved in this case. If, now, $\chi\left(X, \mathcal{O}_{X}\right) \neq 0$, it follows from the discussion above that there exists $p \geq 1$ such that $\mathcal{H}_{(2)}^{0, p}(\tilde{X}) \neq\{0\}$. By conjugation $\mathcal{H}_{(2)}^{p, 0}(\tilde{X}) \neq\{0\}$, hence we get a non-zero $L^{2}$-holomorphic $p$-form for some $p \geq 1$.

The rest of the proof consists, following [9], in constructing from this $L^{2}$ section a non-zero $\Gamma$-invariant section of some $S^{k} \Omega_{\tilde{X}}^{p}$. This can be done using a construction which goes back to Poincaré, that we now describe in a general setting.

. Let $M$ be a complex manifold and $E$ be a holomorphic vector bundle on $M$. Let $\Gamma$ be a countable discrete group acting on $M$ and suppose that the action of $\Gamma$ lifts to an action on $E$. Let $h_{E}$ be a $\Gamma$-invariant continuous hermitian metric on $E$. Let $\Phi: \mathbb{P}(E) \rightarrow M$ denote the projective bundle of hyperplanes in $E$ and $\mathcal{O}_{E}(1) \rightarrow \mathbb{P}(E)$ be the tautological line bundle endowed with the induced hermitian metric $h_{L}$. By functoriality the group $\Gamma$ acts on $\mathbb{P}(E)$ and $\mathcal{O}_{E}(1)$, and all the maps considered above are $\Gamma$-equivariant. As $\Phi_{*}\left(\mathcal{O}_{E}(k)\right)=S^{k} E$ for all $k \geq 1$ (where $\mathcal{O}_{E}(k)$ denotes the line bundle $\mathcal{O}_{E}(1)^{\otimes k}$ ), there is a $\Gamma$-equivariant identification between the space of holomorphic sections $H^{0}\left(\mathbb{P}(E), \mathcal{O}_{E}(k)\right)=H^{0}\left(M, S^{k} E\right)$ under which $L^{q}$ holomorphic sections are identified for all $q \geq 1$.

To any $L^{1}$ holomorphic section $s$ of $E$ we can associate a $\Gamma$-invariant section of $S^{k} E$ for all $k \geq 1$ (the so-called Poincaré series) as follows :

$$
P_{k}(s)(x):=\sum_{\gamma \in \Gamma} \gamma^{*} s^{k}(\gamma \cdot x)
$$


As $s$ is $L^{1}$, this series converges absolutely to a $\Gamma$-invariant holomorphic section of $S^{k} E$.

Moreover, if $s$ is not the zero section, then $P_{k}(s)$ is non-zero for infinitely many $k \geq 1$. Indeed, the precededing construction shows that we need only to consider the case where $E$ is a line bundle. The assertion is then a consequence of the following lemma.

Lemma 2.3. (See Lemma 3.2.A from [9]) Let $\left\{a_{i}\right\}$ be an $l^{1}$-sequence of complex numbers, not all zero. Then there are infinitely many $k \geq 1$ such that $\sum_{i} a_{i}^{k} \neq 0$.

Now recall that in the case where $\chi\left(X, \mathcal{O}_{X}\right) \neq 0$, we showed the existence of a non-zero $L^{2}$ section $s$ of $\Omega_{\tilde{X}}^{p}$ for some $p>0$. If we see $s$ as a section of the tautological line bundle $\mathcal{O}_{\Omega_{\tilde{X}}^{p}}(1)$ on the projectified bundle of $\Omega_{\tilde{X}}^{p}$, then $s^{\otimes k}$ is a non-zero $L^{1}$ section of $\mathcal{O}_{\Omega_{\tilde{X}}^{p}}(1)$ for any $k \geq 2$. Applying the averaging construction just described to $s^{\otimes 2}$, we get a non-zero $\Gamma$-invariant section of some $\mathcal{O}_{\Omega_{\tilde{X}}^{p}}(2 k)$, giving a non-zero section of $S^{2 k} \Omega_{X}^{p}$, as claimed. This concludes the proof.

Remark. For any compact connected Kähler manifold $X$ with infinite fundamental group, let $P(X)$ (resp. $P_{(2)}(X)$ ) be the set of integers $p$ such that $H^{0}\left(X, S^{k} \Omega_{X}^{p}\right) \neq\{0\}$ for some $k>0$ (resp. such that $H_{(2)}^{0}\left(X^{\prime}, S^{k} \Omega_{X^{\prime}}^{p}\right) \neq\{0\}$ for some $k>0$ and some infinite connected étale cover $X^{\prime}$ of $\left.X\right)$. The arguments above show that $P_{(2)}(X) \subset P(X)$. Complex tori show that this inclusion can be strict.

\section{A CRITERION FOR RATIONAL CONNECTEDNESS.}

Recall the following consequence of the 'Abundance Conjecture'

Conjecture. ('uniruledness' conjecture) Let $X$ be a connected compact Kähler manifold. Then $X$ is uniruled (i.e. covered by rational curves) if and only if $H^{0}\left(X, K_{X}^{\otimes k}\right)=\{0\}$ for all $k>0$.

Consider also the following conjecture:

Conjecture. Let $X$ be a connected compact Kähler manifold. Then $X$ is rationally connected (i.e. any two generic points are joined by some rational curve) if and only if $H^{0}\left(X, S^{k} \Omega_{X}^{p}\right)=0$, for every $k>0$ and $p>0$.

\footnotetext{
${ }^{5}$ We thank C. Mourougane for observing that in our first version, our construction appeared to give a section of $S^{k}\left(S^{2}\left(\Omega_{X}^{p}\right)\right)$, instead of $S^{2 k}\left(\Omega_{X}^{p}\right)$.

${ }^{6} \mathrm{~A}$ weaker form, usually attributed to D. Mumford, claims the same conclusion assuming that $H^{0}\left(X,\left(\Omega_{X}^{1}\right)^{\otimes k}\right)=\{0\}$ for all $k>0$.
} 
In [6] a weaker form of Conjecture 3 is established: $X$ is rationally connected if $H^{0}\left(X, S^{k} \Omega_{X}^{p} \otimes A\right)=0$, for every $k>k(A)$, every $p>0$, and some ample line bundle $A$ on $X$.

For both conjectures, the "only if" part is easy. The second conjecture implies theorem 1.1 above, since rationally connected manifolds are simply connected [3].

Let us show that the first conjecture implies the second. First, a Kähler manifold $X$ as in the second conjecture has $h^{2,0}(X)=0$, so it is projective algebraic by Kodaira's projectivity criterion. Now consider the so-called 'rational quotient' $r_{X}: X \rightarrow R$ (constructed in 4 ] and in [11], where it is called the 'MRC'-fibration), which has rationally connected fibres and non-uniruled base $R$ (by [7]). Assuming that $r:=\operatorname{dim}(R)>0$, we get a contradiction, since by the first conjecture there exists a non-zero $s \in H^{0}\left(R, K_{R}^{\otimes k}\right)$, for some $k>0$, which lifts to $X$ as a non-zero section of $H^{0}\left(X, S^{k} \Omega_{X}^{r}\right)$. Thus $r=0$ and $X$ is rationally connected.

Remark. For any compact connected Kähler manifold, let $r^{-}(X):=$ $\max \left\{p \geq 0 \mid \exists k>0, H^{0}\left(X, S^{k} \Omega_{X}^{p}\right) \neq\{0\}\right\}$. Let $r(X):=\operatorname{dim}(R), R$ as above. The preceding arguments show that $r(X) \geq r^{-}(X)$, and the uniruledness conjecture is equivalent to the equality: $r(X)=r^{-}(X)$.

\section{Proof of COROllary 1}

The corollary 1 is an easy consequence of the theorem 1.1 and the following, the proof and statement of which are inspired by [10], theorem 5.2 :

Theorem 4.1. Let $f: X \rightarrow S$ be a proper holomorphic map with connected fibres between connected complex manifolds. Assume that $X$ admits a Kähler metric and that there exists a smooth fibre $X_{s}$ of $f$ which is simply-connected and satisfies $H^{p}\left(X_{s}, \mathcal{O}_{X_{s}}\right)=0$ for all $p>0$. Then $f_{*}: \pi_{1}(X) \rightarrow \pi_{1}(S)$ is an isomorphism of groups.

Proof. First observe that all the smooth fibres $X_{s}$ of $f$ are simplyconnected and satisfy $H^{p}\left(X_{s}, \mathcal{O}_{X_{s}}\right)=0$ for all $p>0$. Indeed, the restriction of $f$ to its smooth locus $S^{o} \subset S$ is topologically a locally trivial fiber bundle by Ehresmann's lemma, and the dimension of $H^{p}\left(X_{s}, \mathcal{O}_{X_{s}}\right)$ is locally constant for $s \in S^{o}$, as follows from the theory of variations of Hodge structures.

Let us first consider the following special case: $X$ is a connected complex Kähler manifold, $f: X \rightarrow \Delta$ is a proper holomorphic map with connected fibres, smooth outside $0 \in \Delta$. Recall that in this situation $X_{0}$ is a retract of $X$. We have to show that the fundamental group of 
$X$ (which is isomorphic to $\pi_{1}\left(X_{0}\right)$ ) is trivial. By blowing-up $X$, one can ensure that $X_{0}$ has only simple normal crossings (i.e. the irreducible components of the corresponding reduced divisor are smooth and meet transversally); this does not change the fundamental group of $X$. By ([10], lemma 5.2.2) the fundamental group of $X$ is finite cyclic, say of order $d$. Let $\pi: \tilde{X} \rightarrow X$ be a universal cover of $X$ and $g: \tilde{X} \rightarrow \Delta$ be the Stein factorization of $f \circ \pi$ so that:

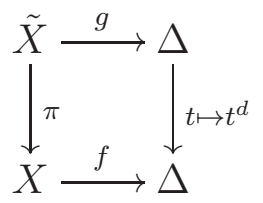

The fibre $\tilde{X}_{t}$ of $g$ at any $t \neq 0$ is isomorphic to $X_{t^{d}}$, hence $H^{p}\left(\tilde{X}_{t}, \mathcal{O}_{\tilde{X}_{t}}\right)=$ $H^{p}\left(X_{t^{d}}, \mathcal{O}_{X_{t^{d}}}\right)=0$ for $t \neq 0$ and $p>0$, and the sheaves $R^{p} g_{*} \mathcal{O}_{\tilde{X}}$ are generically zero for all $p>0$. Being torsion-free (see [16], theorem $\left.2.11^{7}\right)$, they are in fact zero on $\Delta$. Using Leray's spectral sequence, this implies that $H^{p}\left(\tilde{X}, \mathcal{O}_{\tilde{X}}\right)=H^{p}\left(\Delta, g_{*} \mathcal{O}_{\tilde{X}}\right)=0$ for $p>0$. Applying the lemma 4.2 below, it follows that $H^{p}\left(\tilde{X}_{0}^{\text {red }}, \mathcal{O}_{\tilde{X}_{0}^{\text {red }}}\right)=0$ for all $p>0$, hence $\chi\left(\tilde{X}_{0}^{r e d}, \mathcal{O}_{\tilde{X}_{0}^{r e d}}\right)=1$. By multiplicativity of the holomorphic Euler characteristic in finite étale cover (see lemma 2.1), $d=1$ and $X$ is simply-connected.

Lemma 4.2. (Steenbrink, see [17] lemma 2.14 and [10] lemma 5.2.3) Let $X$ be a complex Kähler manifold and let $D \subset X$ be a reduced divisor such that $D$ as a complex space is proper and has normal crossing only. Assume moreover that $D$ is topologically a retract of $X$. Then the restriction maps $H^{p}\left(X, \mathcal{O}_{X}\right) \rightarrow H^{p}\left(D, \mathcal{O}_{D}\right)$ are surjective for all $p \geq 0$.

Proof. Fix a $p \geq 0$. Since $D$ is topologically a retract of $X$, the map $H^{p}(X, \mathbb{C}) \rightarrow H^{p}(D, \mathbb{C})$ is an isomorphism. On the other hand, as $D$ is a union of compact Kähler manifolds crossing transversally, $H^{p}(D, \mathbb{C})$ admits a canonical mixed Hodge structure (see [8] section 4) whose Hodge filtration $H^{p}(D, \mathbb{C})=F^{0} H^{p}(D, \mathbb{C}) \supseteq F^{1} H^{p}(D, \mathbb{C}) \supseteq \cdots$ satisfies $\operatorname{Gr}_{F}^{0} H^{p}(D, \mathbb{C}) \cong H^{p}\left(D, \mathcal{O}_{D}\right)$, see [17] section (1.5). It follows that

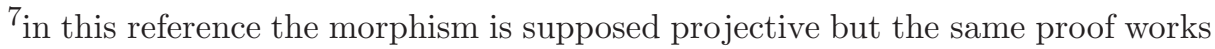
for a proper morphism assuming that the total space admits a Kähler metric. See also [15], corollary 11.18 .
} 
the map $H^{p}(D, \mathbb{C}) \rightarrow H^{p}\left(D, \mathcal{O}_{D}\right)$ is surjective. The following commutative diagram

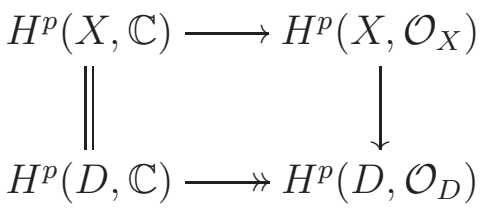

shows that $H^{p}\left(X, \mathcal{O}_{X}\right) \rightarrow H^{p}\left(D, \mathcal{O}_{D}\right)$ is surjective.

We now reduce the general case to this special case. First, because of the following diagram, theorem 4.1 for $f$ follows from the corresponding statement for the restriction of $f$ to an open $U:=S-T$, if the codimension in $S$ of $T$, Zariski closed in $S$, is at least 2:

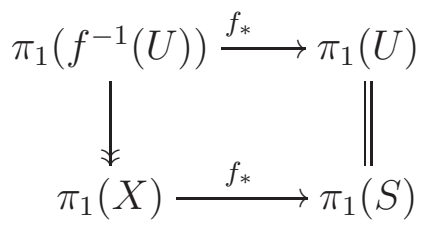

On the other hand, any $s \in S$ admits a contractible neighborhood $U$ in $S$ such that $f^{-1}(U)$ is homeomorphic to $U \times f^{-1}(s)$ (see for example [13]). From this, one easily sees that the theorem 4.1 for $f: X \rightarrow S$ follows if all fibres $X_{s}$ are simply-connected, at least for $s$ outside a codimension $\geq 2$ closed subvariety by the preceding observation.

Let $D \subset S$ be the proper closed subset of points $s$ for which $X_{s}$ is not smooth. By removing a codimension $\geq 2$ subvariety of $S$, one can assume that $D$ is a smooth divisor in $S$. Now, an easy application of Sard's lemma shows that for $s \in D$ outside a proper subvariety $Z \subset D$, there exists a small disk $\Delta_{s}$ crossing $D$ transversally at $s$ such that $f^{-1}\left(\Delta_{s}\right)$ is smooth. For any $s \in D-Z$, the restriction of $f$ to $\Delta_{s}$ satisfies the assumptions of the special case of theorem 4.1 that we showed above, hence $\pi_{1}\left(X_{s}\right)=\pi_{1}\left(f^{-1}\left(\Delta_{s}\right)\right)=\{1\}$.

Let us now explain how the theorems 1.1 and 4.1 imply the corollary 1. First observe that for fixed $k>0$ and $p>0$, the dimension of $H^{0}\left(X_{s},\left(S^{k} \Omega_{X / S}^{p}\right)_{\mid X_{s}}\right)$ is constant on a non empty Zariski open subset of $S$, and this dimension has to be zero by the flat base change theorem. It follows that $H^{0}\left(X_{s}, S^{k} \Omega_{X_{s}}^{p}\right)=0$ for all $k>0$ and $p>0$ for a general smooth fibre $X_{s}$ of $f$. By theorem 1.1 this implies that a general smooth fibre of $f$ is simply connected; hence every smooth fibre is simply connected. The same argument shows that, in particular, for all $p>0, h^{0}\left(X_{s}, \Omega_{X_{s}}^{p}\right)=0$, for $s \in S$ generic, and so: $h^{p}\left(X_{s}, \mathcal{O}_{X_{s}}\right)=0$ 
by Hodge symmetry. We can thus apply theorem 4.1 to conclude the proof of corollary 1 .

\section{REFERENCES}

[1] M. Atiyah. Elliptic operators, discrete groups and von Neumann algebras. Colloque "Analyse et Topologie" en l'Honneur de Henri Cartan (Orsay, 1974), Astérisque, no. 32-33, (1976), 43-72.

[2] Y. Brunebarbe, B. Klingler, and B. Totaro. Symmetric differentials and the fundamental group. Duke Math. J. 162, no.14, (2013), 2797-2813.

[3] F. Campana. On twistor spaces of the class C, J. Differential Geom., 33, no.2, (1991), 541-549.

[4] F. Campana. Connexité rationelle des variétés de Fano. Ann. Sci. Ec. Norm. Sup 25, (1992), 539-545.

[5] F. Campana. Fundamental group and positivity of cotangent bundles of compact Kähler manifolds. J. Algebraic Geom., 4, no.3, (1995), 487-502.

[6] F. Campana, J.P. Demailly, Th. Peternell. Rationally Connected Manifolds and semipositivity of the Ricci Curvature. arXiv:1210.2092.

[7] T. Graber, J. Harris and J. Starr. Families of rationally connected varieties. J. Amer. Math. Soc., 16, no.1, (2003), 57-67.

[8] Ph. Griffiths, W. Schmid. Recent developments in Hodge theory: a discussion of techniques and results. Discrete subgroups of Lie groups and applicatons to moduli (Internat. Colloq., Bombay, 1973), (1975), 31-127.

[9] M. Gromov. Kähler hyperbolicity and $L_{2}$-Hodge theory. J. Differential Geom., 33, no.1, (1991), 263-292.

[10] J. Kollár. Shafarevich maps and plurigenera of algebraic varieties. Inv. Math., 113, (1993), 177-215.

[11] J. Kollár, Y. Miyaoka and S. Mori. Rationally connected varieties. J. Algebraic Geom., 1, no.3, (1992), 429-448.

[12] R. Lazarsfeld. Positivity in algebraic geometry. I Ergebnisse der Mathematik und ihrer Grenzgebiete. 3., 48, (2004).

[13] Le Dung Trang and B. Teissier. Cycles evanescents, sections planes et conditions de Whitney. II [Vanishing cycles, plane sections and Whitney conditions. II] Singularities, Part 2 (Arcata, Calif., 1981), 40, Proc. Sympos. Pure Math., (1983), 65-105.

[14] R. Levy. The Riemann-Roch theorem for complex spaces. Acta Math., 158, no.3-4, (1987), 149-188.

[15] C. Peters and J. Steenbrink. Mixed Hodge structures. Ergebnisse der Mathematik und ihrer Grenzgebiete, 52, Springer-Verlag, (2008).

[16] J. Steenbrink. Mixed Hodge structure on the vanishing cohomology. Real and complex singularities (Proc. Ninth Nordic Summer School/NAVF Sympos. Math., Oslo, 1976), 40, Proc. Sympos. Pure Math., (1977), 525-563.

[17] J. Steenbrink. Mixed Hodge structures associated with isolated singularities. Singularities, Part 2 (Arcata, Calif., 1981), 40, Proc. Sympos. Pure Math., (1983), 513-536.

Yohan Brunebarbe, EPFL, Lausanne, Suisse 
Frédéric Campana, institut Elie Cartan, Université de Lorraine, France, et Institut Universitaire de France 